\title{
Nota de Investigacion/Research Note
}

\section{Perfil de ácidos grasos de Crassostrea rhizophorae proveniente de las lagunas de Chacopata y La Restinga (Venezuela), y el Pantano de Caroni (Trinidad y Tobago)}

\section{Fatty acid profile of Crassostrea rhizophorae from Chacopata and La Restinga lagoons (Venezuela) and Caroni Swamp (Trinidad and Tobago)}

\author{
J Milano¹, H D’Armas*, G Salazar² \\ ${ }^{1}$ Departamento de Química, Escuela de Ciencias, Universidad de Oriente, Núcleo de Sucre, Cumaná, Estado Sucre, Venezuela. \\ *E-mail: haydelba@yahoo.com \\ ${ }^{2}$ Instituto Universitario de Tecnología, Departamento de Química, Cumaná, Estado Sucre, Venezuela.
}

\section{Resumen}

Se evaluó de manera comparativa el contenido de ácidos grasos en los lípidos totales, fosfolípidos y triacilgliceroles de Crassostrea rhizophorae proveniente de las lagunas de Chacopata y La Restinga (Venezuela) y el pantano de Caroni (Trinidad y Tobago). Los fosfolípidos y triacilgliceroles fueron separados de los extractos por cromatografía de capa fina preparativa. La esterificación de los ácidos grasos presentes en estos lípidos seguida de la cromatografía de gases permitió la caracterización y cuantificación de los ácidos grasos presentes en las tres muestras en estudio. Los ácidos grasos saturados fueron los dominantes en los fosfolípidos y triacilgliceroles, seguidos de los ácidos grasos poliinsaturados y, en menor proporción, los ácidos grasos monoinsaturados, a excepción de las muestras provenientes de la Laguna de Chacopata, que presentaron elevadas concentraciones de ácidos grasos poliinsaturados (48.30\%) y mínimas de ácidos grasos saturados (22.70\%) en los triacilgliceroles. El porcentaje total de ácidos grasos poliinsaturados fue mayor en los fosfolípidos que el obtenido para los triacilgliceroles. En los lípidos totales se observaron elevadas concentraciones de ácidos grasos poliinsaturados, seguidas por las ácidos grasos saturados y las menores concentraciones de ácidos grasos monoinsaturados. De acuerdo a las elevadas concentraciones de lípidos y ácidos grasos poliinsaturados encontradas en $C$. rhizophorae en las áreas estudiadas se puede recomendar su consumo para la alimentación de los seres humanos.

Palabras clave: Crassostrea rhizophorae, bivalvo, ácidos grasos, lípidos.

\begin{abstract}
A comparative evaluation was made of the fatty acid content in total lipid, phospholipid and triacylglycerol extracts of Crassostrea rhizophorae, collected from Chacopata and La Restinga lagoons (Venezuela) and Caroni Swamp (Trinidad and Tobago). The phospholipids and triacylglycerols were separated from the extract by preparative thin-layer chromatography. The fatty acid esterification of these lipids followed by gas chromatography allowed the characterization and quantification of the fatty acids present in the three samples studied. The saturated fatty acid content was higher than the polyunsaturated fatty acid content in the phospholipids and triacylglycerols, while the monounsaturated fatty acids occurred in lower proportion, except for the triacylglycerols from the Chacopata sample, which showed high concentrations of polyunsaturated fatty acids (48.30\%) and low concentrations of saturated fatty acids (22.70\%). The total percentage of polyunsaturated fatty acids was higher for phospho-lipids than for triacylglycerols. In the case of total lipids, a high concentration of polyunsaturated fatty acids was observed, followed by saturated fatty acids; monounsaturated fatty acids showed the lowest concentration. Based on the high concentra- tions of lipids and polyunsaturated fatty acids of $C$. rhizophorae found, this species is recommended for human consumption.
\end{abstract}

Key words: Crassostrea rhizophorae, bivalve, fatty acids, lipids.

\section{Introducción}

La acuicultura marina en las últimas décadas ha tenido un auge notable en numerosos países, los cuales están haciendo grandes esfuerzos para lograr que esta actividad sea una fuente confiable de alimentos (Tineo 2002). Sin embargo, cabe mencionar el interés que en la actualidad se tiene en el cultivo de especies comerciales entre las cuales se encuentra la ostra de mangle Crassostrea rhizophorae (Guilding, 1928). Esta

\section{Introduction}

Marine aquaculture has become increasingly important in many countries during the last decades, and great steps are being taken to turn this activity into a safe source of food (Tineo 2002). Special interest in this regard is elicited by commercial species, among them the mangrove oyster Crassostrea rhizophorae (Guilding, 1928). This species has several distinct characteristics, including an extraordinary 
ostra posee particularidades especiales entre las que destacan su alta tasa de crecimiento, alta productividad por área, continua reproducción y propiedades alimenticias que incluyen la fácil digestión de su carne y que sus proteínas son 100\% asimilable (Frías, 1976). La proporción de lípidos y de ácidos grasos en el cuerpo de los animales, entre ellos los moluscos, está íntimamente relacionada con el estado fisiológico del organismo, el crecimiento, su dieta, las reacciones del organismo ante las variaciones del medio, y en general, con todos los factores internos y externos que inciden en cada momento de la vida de cada individuo (Maza 1991).

En Venezuela se han realizado estudios relacionados con la composición química de las ostras. Los primeros datos fueron reportados por Establier (1966) para Crassostrea angulata, quien relacionó la composición con las variaciones hidrográficas y el fitoplancton; Bonilla et al. (1969) determinaron la variación estacional de la composición química de $C$. rhizophorae en Laguna Grande y la Bahía de Mochima, observándo que las proteínas variaron en relación inversa a los elementos de engorde (carbohidratos y grasas), y notando además que los compuestos químicos mostraron valores más elevados en Laguna Grande. Bonilla (1972, 1975) estudió la variación mensual de la composición química en el ostión C. rhizophorae natural. Cavalcanti y Da Cruz (2004) determinaron la composición porcentual del contenido de lípidos y ácidos grasos en $C$. rhizophorae provenientes de Barra de Guaratiba (Río de Janeiro, Brazil), encontrando elevadas concentraciones de lípidos y ácidos grasos poliinsaturados n-3, por lo que la recomendaron para el consumo humano. Muchas de estas investigaciones han sido realizadas para establecer la distribución cuantitativa de los sustratos energéticos de los diferentes tejidos de diversas especies en su ambiente natural, indicando que los cambios en la composición de los nutrientes en los tejidos reflejan la condición fisiológica del organismo asociada con su estado energético, el cual es determinado por interacciones complejas entre los procesos de crecimiento y reproducción, y factores ecológicos tales como los climáticos y la disponibilidad de alimentos.

La salud y el crecimiento corporal de los seres humanos están relacionados con factores ambientales, nutricionales y metabólicos, entre los que los contenidos energéticos, en especial los de ácidos grasos, tienen una gran importancia ya que éstos constituyen una reserva energética y química para el hombre. Por esta razón, el conocer la distribución de los lípidos en el tejido de una especie potencialmente cultivable como $C$. rhizophorae podría definir su valor energético u orientar a las pesquerías comerciales hacia un mayor comercio de esta especie.

Hasta la realización del presente trabajo, en las diversas lagunas de Venezuela no se había estudiado de forma comparativa la composición de ácidos grasos en la ostra de mangle. De allí el interés de evaluar los contenidos de $C$. rhizophorae proveniente de las lagunas de Chacopata y La Restinga (Venezuela) y el pantano de Caroni (Trinidad y Tobago). growth rate, high fecundity per area and uninterrupted reproduction, as well as nutritional properties, such as readily digestible meat and $100 \%$ highly absorbable proteins (Frías 1976). The proportion of lipids and fatty acids in the body of animals, including mollusks, is closely related to the physiological condition of the organism, growth, diet, reactions to environmental variations and, in general, to all the internal and external factors that have a bearing on the life of each individual (Maza 1991).

In Venezuela, extensive research on the chemical composition of oysters has been undertaken. The first data reported were those of Establier (1966), who studied Crassostrea angulata and its relation to hydrographic variations and phytoplankton. Bonilla et al. (1969) determined the seasonal variation of the chemical composition of $C$. rhizophorae populations in Laguna Grande and Mochima Bay, concluding that their protein content shifted in inverse relation to their fattening constituents (carbohydrates and fats) and that the chemical compound values were higher in the former than in the latter. The monthly variation of $C$. rhizophorae would later be studied by Bonilla (1972, 1975). Cavalcanti and Da Cruz (2004) determined the percentage composition of the lipid and fatty acid content of $C$. rhizophorae from Barra de Guaratiba, in Rio de Janeiro, Brazil, and found elevated concentrations of lipids and n-3 polyunsaturated fatty acids, which prompted them to recommend this species for human consumption. Much of this research has been undertaken to establish the quantitative distribution of energy substrata in the various tissues of different species in their natural environment, since changes in the nutritional makeup of the tissues reflect the physiological condition of the organisms in association with their energy status, which is determined by the complex interactions governing growth processes, reproductive activities and ecological factors such as the weather and food availability.

The well-being and physical development of individuals are related to environmental, nutritional and metabolic constituents, the energy contents, especially the fatty acids, having a tremendous importance on account of their energy and chemical storage capacity in humans. Knowledge of the lipid distribution in the tissue of a potentially farmable species could define the energy strength in that species. In the case of $C$. rhizophorae, this information might lead to greater commercialization of this species.

Prior to this research, no comparative study had been carried out of the fatty acid composition in mangrove oyster populations from different lagoons in Venezuela. This investigation undertakes a comparison of the fatty acid content in $C$. rhizophorae samples from Chacopata and La Restinga lagoons, in Venezuela, and Caroni Swamp, in Trinidad and Tobago.

\section{Material and methods}

\section{Study area}

Chacopata Lagoon is located to the north of the Araya Peninsula, in the state of Sucre, Venezuela. The lagoon mouth 


\section{Materiales y métodos}

\section{Área de muestreo}

La Laguna de Chacopata está situada al norte de la Península de Araya (Estado Sucre, Venezuela); su boca se encuentra en comunicación con el Mar Caribe. El suministro de agua dulce a esta laguna es aportado por algunos arroyos de escaso caudal y su perímetro costero presenta formaciones de manglares. Además de abundantes manglares, esta región está sujeta a vientos torrenciales, fuertes oleajes, elevada salinidad y altas temperaturas creando condiciones estables que permiten la fijación de larvas, el crecimiento y engorde de las ostras.

El Parque Nacional Laguna de La Restinga está constituido por una estrecha franja de arena, que une la parte oriental de la Isla Margarita con la Península de Macanao (Estado Nueva Esparta, Venezuela), y una gran laguna costera donde se ha consolidado una comunidad de manglares. La laguna tiene un lecho de más de $100 \mathrm{~km}^{2}$ y extensas áreas de manglares que dan origen a gran número de islas y canales. Sus playas sobre el océano están formadas por depósitos de conchas fósiles pulverizadas debido a la acción del oleaje. La vegetación predominante está representada por manglares donde destacan el mangle rojo, el negro y el blanco. Los vientos en la zona son débiles, y el agua de la laguna es turbia y con salinidad moderada.

El Parque Pantano de Caroni se encuentra al sur de Puerto España (Trinidad y Tobago) y tiene una extensión de 56,110 $\mathrm{m}^{2}$. Se considera uno de los mejores ejemplos de complejo ecosistema pantanal de manglares, aguas dulces y salinas, vientos moderados, baja salinidad y aguas fangosas.

\section{Muestreo}

Las ostras de mangle fueron recolectadas en las lagunas de Chacopata y La Restinga (Venezuela) y el pantano de Caroni (Trinidad) durante agosto de 2001, tiempo que coincide con el desove de la especie y la incidencia de mayores tallas. La temperatura del agua en cada área de estudio fue medida con un termómetro de mercurio y la salinidad con un refractómetro (Aquafauna, Bio-Marine, USA). En cada área de estudio se recolectó un conjunto de 60 ostras con talla de 5 a $6 \mathrm{~cm}$. Se hicieron tres réplicas con cada conjunto. Se transportaron al laboratorio en una hielera con hielo seco. Se les extrajo el tejido blando, se limpiaron y lavaron con agua desionizada para eliminar la mayor cantidad de sales, iones y fitoplancton presentes y se congelaron a $-20^{\circ} \mathrm{C}$ para su posterior análisis en el laboratorio.

\section{Análisis de los lípidos}

Para la extracción de los lípidos, previamente se realizó la maceración del tejido blando. Se tomó $1 \mathrm{~g}$ del material triturado y se le agregó $20 \mathrm{~mL}$ de una mezcla de $\mathrm{CHCl}_{3}: \mathrm{CH}_{3} \mathrm{OH}$ (2:1) como solvente para cada muestra (Overturf y Dryer opens to the Caribbean Sea. Scant-flow streams supply fresh water to the lagoon, whose coastal fringes are festooned by dense mangrove patches that, together with gusty winds, strong waves, heightened salinity and high temperatures, create stable conditions for larval settlement, spat growth and fattening.

La Restinga Lagoon National Park, in the state of Nueva Esparta, Venezuela, consists of a narrow strip of sand that joins the eastern part of Margarita Island to the Macanao Peninsula. This mangrove-filled and stretched-out lagoon features a bed of over $100 \mathrm{~km}^{2}$, the mangrove forming a great number of islets and waterways. The oceanic front beaches abound with fossil shells pulverized by the action of the waves. The predominant vegetation is the mangrove, in its red, black and white variations. The lagoon's muddy waters feature moderate salinity and are combed by frail winds.

Caroni Swamp covers an area of $56,110 \mathrm{~m}^{2}$. It is located to the south of Port of Spain in Trinidad. It is considered one of the best models of swampy mangrove ecosystems, with both fresh- and salt-water supplies, moderate winds, low salinity and muddy waters.

\section{Sampling}

Sampling was carried out in a series of spots representing each area of the lagoons. The mangrove oysters were collected from the Chacopata and La Restinga lagoons and Caroni Swamp in August 2001, at the onset of the spawning season, when the specimens have the largest commercial size. Water temperature at each site was recorded with a mercury thermometer. Water salinity was checked with a refractometer (Aquafauna, Biomarine, USA). A 60-oyster sample, each specimen ranging from 5 to $6 \mathrm{~cm}$, was collected in each study area. Three subgroups were assembled from this sample, placed in a cooler containing dry ice and transported to the laboratory, where the soft tissue was removed, cleaned, rinsed with deionized water to get rid of salts, ions and phytoplankton, and frozen at $-20^{\circ} \mathrm{C}$ for further analysis.

\section{Lipid analysis}

Prior to lipid extraction, the soft tissue was mashed down. One gram of the ground material was macerated in $20 \mathrm{~mL}$ of a 2:1 mixture of $\mathrm{CHCl}_{3}: \mathrm{CH}_{3} \mathrm{OH}$ (Overturf and Dryer 1967). The tissue, along with the solvent mixture, was stirred for $1 \mathrm{~h}$ and filtered through GF/C grade Whatman paper (No. 6), and the residue thus obtained was submitted to a second and similar extraction procedure to secure an effective and complete lipid removal.

The combined filtrate containing the lipids was placed in a separation funnel, and a saturated solution of $\mathrm{NaCl}$ amounting to nearly one third of the filtrate volume was added to it. The resulting filtrate was stirred several times and stored under refrigeration for approximately $16 \mathrm{~h}$, after which anhydrous $\mathrm{Na}_{2} \mathrm{SO}_{4}$ was added as a water-absorbing agent once the organic phase had been extracted. The largest amount of solvent 
1967). El tejido con la mezcla de solventes se sometió a agitación continua por una hora, se filtró con papel de filtro Whatman $\mathrm{N}^{\circ} 6$, tipo GF/C y el residuo fue extraído nuevamente, utilizando el mismo procedimiento para garantizar la extracción eficaz y completa de los lípidos.

El filtrado combinado que contenía los lípidos se colocó en un embudo separador y se le agregó una solución saturada de cloruro de sodio $(\mathrm{NaCl})$, en una proporción equivalente a aproximadamente un tercio del volumen del filtrado. Se agitó varias veces y se guardó en refrigeración por 16 h, aproximadamente. Se extrajo la capa orgánica, se le agregó $\mathrm{Na}_{2} \mathrm{SO}_{4}$ anhidro como absorbente del agua y se evaporó la mayor cantidad de mezcla de solventes en un rotoevaporador. Luego, a la fracción lipídica obtenida se le burbujeó nitrógeno gaseoso y se refrigeró, cuantificando los lípidos totales por gravimetría.

Los lípidos se separaron en sus diferentes clases mediante cromatografía de capa fina preparativa (CCFP). Las placas se prepararon utilizando silica gel DG 60 como adsorbente y en capas de $1.0 \mathrm{~mm}$ de espesor. El extracto lipídico se disolvió en $\mathrm{CHCl}_{3}$ y se colocó en las placas cromatográficas. El desarrollo se realizó utilizando una mezcla de éter de petróleo-éter dietílico-ácido acético (87:12:1) como solvente. Los lípidos se identificaron comparando los factores de retención, $\mathrm{R}_{\mathrm{f}}$ (razón de la distancia recorrida por el soluto entre la distancia recorrida por el solvente), de las distintas bandas obtenidas con los de patrones comerciales de Sigma Chemical Co. Se aislaron las bandas correspondientes a triacilgliceroles y fosfolípidos, recuperándolos con éter dietílico y metanol, respectivamente (Carter 1993).

Los ésteres metílicos de estos lípidos se obtuvieron a través del método de Brokerchoff (Cristie 1973), que consiste en saponificar entre 20 y 40 mg de muestra lipídica disolviéndola en $0.5 \mathrm{~mL}$ de éter dietílico, añadiendo $1.0 \mathrm{~mL}$ de $\mathrm{KOH} 0.5 \mathrm{~mol}$ $\mathrm{L}^{-1}$ en metanol (seco) y dejando en reposo por $10 \mathrm{~min}$. Una vez transcurrido este tiempo se agregó $0.5 \mathrm{~mL}$ de $\mathrm{HCl} 1.0 \mathrm{~mol} \mathrm{~L}^{-1} \mathrm{y}$ posteriormente se extrajeron los ésteres metílicos de los ácidos grasos con $3.0 \mathrm{~mL}$ de éter de petróleo, en tres porciones de 1.0 $\mathrm{mL}$. Finalmente se reunieron los extractos de ésteres metílicos y se evaporó el solvente hasta sequedad con nitrógeno gaseoso (Satue et al. 1994).

Se empleo cromatografía gas-líquido para determinar la composición porcentual de ácidos grasos en las muestras lipídicas. Los ésteres metílicos correspondientes a cada muestra se analizaron en un cromatógrafo Agilent modelo 4890 D acoplado a un detector de ionización de llama (FID) y una columna HP-INNOWax 30 m 0.32 mm ID, fase líquida. Se usó nitrógeno como gas de arrastre a un flujo de $1.8 \mathrm{~mL} \mathrm{~min}^{-1}$. La separación se realizó en las siguientes condiciones: temperatura del inyector: $300^{\circ} \mathrm{C}$; temperatura del detector: $300^{\circ} \mathrm{C}$; y temperatura de la columna: $200^{\circ} \mathrm{C}$. El área de los picos se determinó con un sistema de adquisición de datos HP 3398AGC Chemstation acoplado al cromatógrafo. La identificación de los ácidos grasos se llevó a cabo mediante comparación de los tiempos de retención determinados con los de patrones comerciales de ésteres metílicos y los resultados se mixture was evaporated in a rotating evaporator and the lipid fraction obtained was bubbled with gaseous nitrogen, refrigerated, and its total lipids quantified by gravimetric analysis.

The lipids were divided into different categories by preparative thin layer chromatography. The plates were prepared using silica gel DG60 as an adsorbent and arranged in 1-mmthick layers. The lipid extract was dissolved in $\mathrm{CHCL}_{3}$ and placed onto the chromatography plates. An 87:12:1 mixture of petroleum ether, diethyl ether and acetic acid was used as a solvent front to develop the chromatogram. The lipids were identified comparing the retention factors $\left(\mathrm{R}_{\mathrm{f}}\right.$, or ratio of the run length of the solute to the distance reached by the eluent) of the different bands obtained with Sigma Chemical Co. commercial patterns. The bands corresponding to the triacylglycerols and phospholpids were isolated, these compounds being recovered with diethyl ether and methanol, respectively (Carter 1993).

The methyl esters of these lipids were obtained by the Brokerchoff method (Cristie 1973), which consisted of saponifying a fraction of $20-40 \mathrm{mg}$ of lipids, dissolving it in $0.5 \mathrm{~mL}$ of diethyl ether, adding $1.0 \mathrm{~mL} \mathrm{KOH} 0.5 \mathrm{~mol} \mathrm{~L}^{-1}$ in dry methanol and letting it sit for $10 \mathrm{~min}$, after which $0.5 \mathrm{~mL} \mathrm{HCl}$ $1.0 \mathrm{~mol} \mathrm{~L}^{-1}$ was added. The fatty acid methyl esters were subsequently extracted with three portions of $1.0 \mathrm{~mL}$ of petroleum ether; the methyl ester extracts were brought together and the solvent was evaporated to dryness with gaseous nitrogen (Satue et al. 1994).

Liquid gas chromatography was used to determine the fatty acid percentage composition in the lipid samples. The methyl esters corresponding to each sample were analyzed in an Agilent 4890 D liquid-gas chromatograph coupled to a flame ionization detector and a 30-m, 0.32-mm ID liquid phase HPINNOWax column. Nitrogen was used as carrier gas at a flow rate of $1.8 \mathrm{~mL} \mathrm{~min}{ }^{-1}$. The separation was carried out under the following conditions: injector temperature of $300^{\circ} \mathrm{C}$, detector temperature of $300^{\circ} \mathrm{C}$ and column temperature of $200^{\circ} \mathrm{C}$. The peak area was determined with an HP3398AGC Chemstation data acquisition system coupled to the chromatograph. Fatty acid identification was carried out by comparing the determined retention times with those of the methyl ester commercial patterns, and the results were expressed as percentages of the total of fatty acids present in the lipid samples for each locality (Farnworth et al. 1982).

\section{Statistical analysis}

The data were run through a simple variance analysis (single factor ANOVA) to determine the significant differences in the content of lipids and fatty acids in the samples studied. All the statistical analyses were obtained using the Statgraphics Plus 4.1 statistical software package. A value of $P<0.05$ was taken as a significant difference for the analyses and the data were corroborated through an a posteriori LSD test to determine the existing groups. 
expresaron en porcentajes del total de ácidos grasos presentes en las muestras lipídicas de cada localidad (Farnworth et al. 1982).

\section{Análisis estadísticos}

Los datos fueron analizados estadísticamente por un análisis de varianza sencillo (ANOVA de un factor) para determinar las diferencias significativas en el contenido de lípidos y ácidos grasos en las muestras estudiadas. Todos los análisis estadísticos fueron obtenidos usando el paquete estadístico Statgraphics Plus 4.1 con un valor de $P<0.05$ como diferencia significativa para los análisis, y los datos fueron constatados a través de una prueba a posteriori (LSD) para determinar los grupos existentes.

\section{Resultados y discusión}

\section{Lípidos totales}

La mayor concentración de lípidos (tabla 1) se obtuvo en las ostras provenientes de la Laguna de Chacopata con un valor de $7.55 \pm 1.3 \%$, con diferencias altamente significativas $(P<$ 0.05 ) en el contenido lipídico de las muestras de estudio. Esto se constató a través de la prueba a posteriori (LSD), que mostró la formación de tres grupos: Laguna de Chacopata (7.55 \pm 1.3\%), Pantano de Caroni (3.69 $\pm 0.09 \%)$ y La Restinga (2.71 $\pm 0.01 \%)]$.

En esta investigación se observó un gran potencial energético en las ostras de la Laguna de Chacopata, en comparación con las de La Restinga y el Pantano de Caroni. Estas diferencias en el contenido pudo deberse a cambios bruscos en las condiciones ambientales de cada una de las áreas en estudio. En el mes de muestreo la Laguna de Chacopata presentó una salinidad de $42 \%$, temperaturas entre $29^{\circ} \mathrm{C}$ y $30^{\circ} \mathrm{C}$, vientos fuertes y agua turbias, condiciones pudieron permitir una fácil adaptación de las poblaciones aumentando la capacidad de los ostiones para ajustar su metabolismo, favoreciendo así a una elevada producción de grasas que serían utilizada para su reproducción. A diferencia de las otras áreas estudiadas, donde la salinidad fue de alrededor de $37 \%$, la temperatura entre $27^{\circ} \mathrm{C}$ y $29^{\circ} \mathrm{C}$, con vientos moderados y aguas fangosas, presentando condiciones menos favorables para la formación de grasas en la especie. En consecuencia, las ostras de la Laguna de Chacopata lograron una mayor actividad alimenticia, aprovechando mejor el suministro de plancton y sales nutritivas, así como los recursos energéticos para la reproducción y ajustando su metabolismo a las condiciones dominantes en la zona (altas temperaturas, vientos fuertes y elevada salinidad, entre otros), tal como lo señala Bonilla (1975).

\section{Análisis de los ácidos grasos en los lípidos totales}

En los lípidos totales (tabla 2) los ácidos grasos dominantes fueron los poliinsaturados con valores mínimos de $49.0 \%$ en

\section{Results and discussion}

\section{Total lipids}

The largest concentration of lipids $(7.55 \pm 1.3 \%)$ was recorded for the Chacopata oysters and highly significant differences $(P<0.05)$ were observed. The results were confirmed by the a posteriori test applied, which validated the three lipid concentration groups, namely, Chacopata Lagoon, Caroni Swamp and La Restinga Lagoon, with values of $7.55 \pm 1.3 \%$, $3.69 \pm 0.09 \%$ and $2.71 \pm 0.01 \%$, respectively (table 1 ).

This research revealed a high energetic potential on the part of the Chacopata oysters, as opposed to those from La Restinga and Caroni. This contrast may be due to abrupt changes in the weather conditions of each study area. In August 2001, Chacopata Lagoon had $42 \%$ salinity, temperatures of $29-30^{\circ} \mathrm{C}$, strong winds and muddy waters, conditions that allowed the oysters to adjust their metabolism accordingly, resulting in an increased production of fat for reproductive purposes. The other areas had conditions less likely to elicit fat production by this species. Thus, the Chacopata oysters showed more vigorous nutritional activity and took better advantage of the plankton and nutritious salt supply required for procreation, just as reported by Bonilla (1975).

\section{Analysis of fatty acids in total lipids}

Polyunsaturated fatty acids were predominant in the total lipids (table 2 ), the minimum value (49.0\%) corresponding to the La Restinga samples and the maximum (54.3\%) to the Chacopata oysters. The prevailing fatty acids were adrenic acid (22:4 n-3), with proportions ranging from $31.2 \%$ to $37.2 \%$, followed by eicosenoic acid (20:2 n-3), ranging from $6.9 \%$ to

Tabla 1. Porcentajes de lípidos totales de Crassostrea rhizophorae proveniente de las diferentes zonas en estudio.

Table 1. Percentage of total lipids in Crassostrea rhizophorae from the different study areas.

\begin{tabular}{lccc}
\hline Muestras & $\begin{array}{c}\text { Réplicas } \\
\text { \% lípidos }\end{array}$ & $\overline{\mathrm{X}}$ & $\mathrm{S}$ \\
\hline Laguna de Chacopata & 6.22 & $7.55^{*}$ & 1.29 \\
& 8.79 & & \\
& 7.65 & & \\
Laguna de La Restinga & 2.70 & $2.71^{*}$ & 0.01 \\
& 2.71 & & \\
Pantano de Caroni & 2.72 & & \\
& 3.60 & $3.69 *$ & 0.09 \\
& 3.78 & & \\
\hline
\end{tabular}

${ }^{*} P<0.05$, medias con diferencias significativas.

$\mathrm{N}=3$, números de réplicas (homogenizado de pools de 60 ostras).

$\mathrm{S}=$ desviación estándar. 
las muestras de La Restinga, y máximos de 54.3\% en las de Chacopata. Entre los ácidos grasos presentes prevalecen el ácido adrénico (22:4 n-3), entre 31.2\% y 37.2\%, seguido por el eicosadoenoico (20:2 n-3), entre 6.9\% y 7.4\%, el docosapentaenoico (22:5 n-3), el eicosapentaenoico (20:5 n-3) y el octadecatrienoico (18:3 n-3) en menores proporciones. Estos valores son tres veces superiores a los encontrados por Cavalcanti y Da Cruz (2004) y Noffs (2002). Estas divergencias pudieran deberse a las diferentes condiciones ambientales presentes en las zonas de muestreo dado que en sus estudios esos autores demostraron que a medida que aumenta la temperatura en un área, la concentración de los ácidos grasos poliinsaturados también se incrementa.

Los análisis estadísticos realizados no evidenciaron diferencias significativas $(P>0.05)$ en las concentraciones de ácidos grasos saturados, poliinsaturados y monoinsaturados entre las muestras estudiadas. Debido al elevado contenido de estos ácidos grasos se puede sugerir que el consumo humano de esta especie es beneficioso para la prevención de enfermedades aterioscleróticas, tal y como se reporta en diversos estudios que demuestran que el consumo de ácidos grasos poliinsaturados reducen los niveles de colesterol asociado a lipoproteínas de baja densidad (LDL) y, por ende, disminuyen la presión sanguínea y la viscosidad de la sangre (Cardona y Soltero 1987, Vakkilainen et al. 2002). De allí que su consumo podría disminuir el riesgo a sufrir enfermedades cardiovasculares y cumplir una acción antitrombótica y antiaterogénica en los seres humanos (Carmena 1986, Pieter et al. 1994, Cohn et al. 2002).

Las grasas saturadas constituyeron entre $25.9 \%$ y $28.8 \%$ del total de lípidos, observándose valores mínimos de $25.9 \%$ en las muestras procedentes del Pantano de Caroni, y máximos de $28.8 \%$ en las de las lagunas de La Restinga y Chacopata. Los ácidos grasos saturados más abundantes fueron el ácido palmítico (16:0) con $11.7 \%$ en las muestras de La Restinga, 9.0\% en las del Pantano de Caroni y 6.1\% en las de Chacopata, seguido del lignócerico (24:0) con valores mínimos de 3.0\% en las ostras de La Restinga y máximo en las de Chacopata (11.2\%); el ácido mirístico (14:0) y el esteárico (18:0) mostraron los menores valores para los ácidos grasos saturados. Las elevadas concentraciones observadas de estos ácidos grasos podrían sugerir que el consumo de estos organismos, dada su abundancia en estas grasas, incrementaría la agregación plaquetaria y aceleraría el proceso de trombosis; sin embargo, estas concentraciones no son preocupantes si se comparan con las elevadas concentraciones de ácidos grasos poliinsaturados obtenidas en las diferentes muestras, por las cuales se podría inferir que el consumo frecuente de esta especie posiblemente sería capaz de impedir el desarrollo de ateromas y el depósito de colesterol en el organismo humano (Cardona y Soltero 1987, Goldbourt y Yaari 1990). Los ácidos grasos monoinsaturados se presentaron en menores proporciones, con valores entre $3.0 \%$ y $5.5 \%$.

Por otro lado, diversas investigaciones realizadas han señalado que los ácidos poliinsaturados del tipo n-3 como el ácido
7.4\%, and docosapentaenoic acid (22:4 n-3), eicosapentaenoic acid (20:5 n-3) and octadecatrienoic acid (18:3n-3) in smaller proportions. These values are three times higher than those found by Noffs (2002) and Cavalcanti and Da Cruz (2004). This divergence might reflect the different environmental

Tabla 2. Composición porcentual de los ácidos grasos en los lípidos totales de Crassostrea rhizophorae proveniente de las lagunas de Chacopata y La Restinga y el Pantano de Caroni (AGS, ácidos grasos saturados; AGMI, ácidos grasos monoinsaturados, AGPI, ácidos grasos poliinsaturados; AGNI, ácidos grasos no identificados).

Table 2. Fatty acid percentages for total lipids in Crassostrea rhizophorae from Chacopata and La Restinga lagoons and Caroni Swamp (AGS, saturated fatty acids; AGMI, monounsaturated fatty acids, AGPI, polyunsaturated fatty acids; AGNI, unidentified fatty acids).

\begin{tabular}{|c|c|c|c|}
\hline Ácidos grasos & $\begin{array}{c}\text { Chacopata } \\
\text { (media } \pm 0.1)\end{array}$ & $\begin{array}{l}\text { La Restinga } \\
\text { (media } \pm 0.1 \text { ) }\end{array}$ & $\begin{array}{c}\text { Caroni } \\
(\text { media } \pm 0.1)\end{array}$ \\
\hline C 8:0 & 1.0 & nd & 0.7 \\
\hline C 10:0 & 1.0 & nd & 0.7 \\
\hline C $14: 0$ & 3.4 & 5.0 & 3.5 \\
\hline C $16: 0$ & 6.1 & 11.7 & 9.0 \\
\hline C 17:0 & 1.0 & 1.5 & nd \\
\hline C 18:0 & 1.9 & 3.1 & 2.6 \\
\hline C 19:0 & 1.3 & 2.0 & nd \\
\hline C 20:0 & 1.2 & 1.9 & nd \\
\hline C 23:0 & 0.7 & 0.7 & 0.6 \\
\hline C 24:0 & 11.2 & 3.0 & 8.8 \\
\hline Total AGS & $28.8 *$ & $28.9 *$ & $25.9 *$ \\
\hline C $16: 1$ & 0.6 & 1.9 & 1.1 \\
\hline C 18:1 n-3 & 0.9 & 1.2 & 0.9 \\
\hline C 18:1 n-6 & 0.8 & 1.4 & 1.2 \\
\hline C 20:1 n-3 & 0.7 & 1.0 & 2.2 \\
\hline Total AGMI & $3.0 *$ & $5.5^{*}$ & $5.4^{*}$ \\
\hline C 18:2 n-6 & 0.8 & nd & 0.6 \\
\hline C 18:3 n-3 & 2.0 & 0.7 & 2.4 \\
\hline C 20:2 n-3 & 6.9 & 7.4 & 7.4 \\
\hline C $20: 3 n-3$ & 0.6 & 1.0 & 0.9 \\
\hline C $20: 5$ n-3 & 1.7 & 1.8 & 2.1 \\
\hline C 22:4 n-3 & 37.2 & 33.8 & 31.2 \\
\hline C 22:5 n-3 & 3.6 & 2.8 & 2.9 \\
\hline C 22:6 n-3 & 1.5 & 1.5 & 1.7 \\
\hline Total AGPI & $54.3^{*}$ & $49.0 *$ & $49.2 *$ \\
\hline Total AGNI & 13.9 & 16.6 & 19.5 \\
\hline
\end{tabular}

$\mathrm{N}=3$, números de réplicas (conjuntos de 60 ostras).

nd: no detectado.

Valores encontrados $=($ medias \pm 0.1$)$.

Límite de detección $\geq 0.1$.

${ }^{*} P>0.05$, no hubo diferencias significativas. 
adrénico (22:4 n-3) y el ácido eicosapentaenoico (20:5 n-3) se encuentran en todas las especies marinas, estableciéndose que el consumo de estos ácidos grasos puede reducir los factores de riesgo relacionadas a lesiones articulares como la ateriosclerosis y la trombosis (Goldbourt y Yaari 1990, Cohn et al. 2002).

\section{Análisis de los ácidos grasos en los fosfolípidos}

En los fosfolípidos (tabla 3) dominaron los ácidos grasos saturados, con valores mínimos (42.1\%) en las ostras provenientes del Pantano de Caroni y máximos (46.8\%) en las La Restinga. Prevalecieron el ácido palmítico (16:0), con valores entre $12.3 \%$ y $15.5 \%$, seguido por el mirístico (14:0) y el esteárico (18:0), los cuales a pesar de presentar elevadas concentraciones en las ostras de Chacopata y La Restinga, no se detectaron en las procedentes del Pantano de Caroni. Al comparar estos resultados con los encontrados por Martino et al. (2002) y Cavalcanti y Da Cruz (2004), se observa que las concentraciones de grasas saturadas encontradas en esta investigación son doblemente superiores a las señaladas por estos autores en sus estudios. Este incremento se atribuye a las elevadas temperaturas observadas en las zonas analizadas, las cuales propician un mayor flujo a través de la membrana celular de los organismos de esta especie y, por ende, el aumento de estos ácidos grasos en los fosfolípidos.

Los análisis estadísticos realizados no evidenciaron diferencias significativas $(P>0.05)$ en las concentraciones de ácidos grasos saturados, poliinsaturados y monoinsaturados en las muestras estudiadas. Los elevados valores de grasas saturadas en el contenido de lípidos podrían sugerir que el exceso en la ingesta de éstos incrementaría los niveles de colesterol en la sangre, especialmente los de la fracción LDL. Aunque aun no se conoce bien el mecanismo por el que se produce este aumento, diversos estudios epidemiológicos (Abbey et al. 1990, Roberts 1992, Cohn et al. 2002, Vakkilainen et al. 2002) indican que estos ácidos grasos enriquecen los fosfolípidos de la membrana celular, interfiriendo así en la función normal de los receptores LDL y reducen la absorción de las LDL en las células, por lo que al disminuir las LDL la concentración de colesterol disminuye. De allí que estas concentraciones de grasas saturadas en los fosfolípidos puedan reducir la formación de estas lipoproteínas de elevada peligrosidad para el cuerpo humano.

Los ácidos grasos poliinsaturados presentaron valores entre $25.2 \%$ y $27.3 \%$, observándose los mínimos en las ostras de la Laguna de Chacopata y los máximos en las de La Restinga. Predominó el ácido adrénico (22:4 n-3), entre 7.0\% y 7.7\%; seguido del docosahexaenoico (22:6 n-3), el linolénico (18:3 n-3) y el docosapentaenoico (20:5 n-3). El elevado contenido de ácidos grasos poliinsaturados mostrado por las ostras provenientes de las diferentes áreas en estudio sugiere que el consumo de estos ácidos grasos podría ser utilizado como almacen de energía en los seres humanos y servir como fuente alimenticia (Satue et al. 1994). conditions at the sampling areas. Studies have demonstrated that the higher the temperature of an area, the higher the concentration of polyunsaturated fatty acids.

The statistical analyses carried out reflected nonsignificant differences $(P>0.05)$ in the concentrations of the saturated, polyunsaturated and monounsaturated fatty acids of the samples under study. Because of the high fatty acid content of C. rhizophorae, it can be suggested that its consumption could prevent atherosclerotic diseases, as has been reported in different studies demonstrating that a diet rich in polyunsaturated fatty acids reduces low density lipoprotein cholesterol levels (LDL) and, therefore, reduces blood pressure and blood viscosity (Cardona and Soltero 1987, Vakkilainen et al. 2002). It can be concluded that oyster intake could diminish the risk of cardiovascular disease and enhance antithrombotic and antiatherogenic activities in human beings (Carmena 1986, Pieter et al. 1994, Cohn et al. 2002).

Furthermore, several studies have pointed out that n-3-type polyunsaturated acids such as adrenic acid (22:4 n-3) and eicosapentaenoic acid (20:5 n-3) are found in all marine species, and that the consumption of these fatty acids could reduce risk factors associated with vascular lesions, such as atherosclerosis and thrombosis (Goldbourt and Yaari 1990, Cohn et al. 2002).

Saturated fats ranged from 25.9\%, in Caroni oysters, to 28.8\%, in La Restinga and Chacopata specimens. The most abundant saturated fatty acids found were palmitic acid (16:0), with values of $11.7 \%, 9.0 \%$ and $6.1 \%$ for La Restinga, Caroni and Chacopata spat populations, respectively, and lignoceric acid (24:0), with values ranging between a minimum of 3.0\% in La Restinga oysters and a maximum of $11.2 \%$ in Chacopata specimens. The least abundant were myristic acid (14:0) and estearic acid (18:0). The elevated concentrations of these fatty acids suggest that a diet rich in these fats may increase platelet aggregation and thrombosis; however, when compared with the high concentrations of polyunsaturated fatty acids obtained from the different samples, these saturated concentrations are not worrisome, and it could be inferred that the frequent consumption of these species might deter atheroma development and cholesterol deposition in the human body (Cardona and Soltero 1987, Goldbourt and Yaari 1990). Monounsaturated fatty acids occurred in smaller proportions, with values of $3.0-5.5 \%$.

\section{Analysis of phospholipid fatty acids}

Among the phospholipids (table 3), the saturated fatty acids predominated, the minimum (42.1\%) and maximum (46.8\%) values corresponding to the Caroni and La Restinga oysters, respectively. Palmitic acid (16:0) was prevalent, with values between $12.3 \%$ and $15.5 \%$, followed by myristic acid (14:0) and estearic acid (18:0); the latter two had elevated concentrations in the Chacopata and La Restinga spat populations but was undetected in the Caroni oysters. A comparison of these results with those of Martino et al. (2002) and Cavalcanti and 
Tabla 3. Composición porcentual de los ácidos grasos en los fosfolípidos de Crassostrea rhizophorae proveniente de las lagunas de Chacopata y La Restinga y el Pantano de Caroni (AGS, ácidos grasos saturados; AGMI, ácidos grasos monoinsaturados, AGPI, ácidos grasos poliinsaturados; AGNI, ácidos grasos no identificados).

Table 3. Fatty acid percentages for phospholipids in Crassostrea rhizophorae from Chacopata and La Restinga lagoons and Caroni Swamp (AGS, saturated fatty acids; AGMI, monounsaturated fatty acids, AGPI, polyunsaturated fatty acids; AGNI, unidentified fatty acids).

\begin{tabular}{|c|c|c|c|}
\hline Ácidos grasos & $\begin{array}{c}\text { Chacopata } \\
\text { (media } \pm 0.1 \text { ) }\end{array}$ & $\begin{array}{l}\text { La Restinga } \\
\text { (media } \pm 0.1 \text { ) }\end{array}$ & $\begin{array}{c}\text { Caroni } \\
(\text { media } \pm 0.1)\end{array}$ \\
\hline C 8:0 & 1.1 & 1.4 & 4.6 \\
\hline C 9:0 & nd & nd & 1.2 \\
\hline C 10:0 & 0.8 & 2.1 & 5.0 \\
\hline C 14:0 & 4.7 & 7.8 & 8.8 \\
\hline C 15:0 & 0.8 & nd & 0.9 \\
\hline C 16:0 & 15.5 & 15.1 & 2.6 \\
\hline C 17:0 & 1.1 & nd & nd \\
\hline C 18:0 & 4.0 & 5.4 & 3.2 \\
\hline C 19:0 & 7.7 & 4.3 & nd \\
\hline C 20:0 & 4.3 & 3.5 & 1.7 \\
\hline C 23:0 & 0.9 & 4.1 & 2.1 \\
\hline C $24: 0$ & 2.1 & 3.1 & 2.3 \\
\hline Total AGS & 43.0* & $46.8 *$ & $42.1 *$ \\
\hline C 16:1 & 1.0 & 1.1 & 0.9 \\
\hline C 18:1 n-3 & 1.4 & 1.7 & 1.5 \\
\hline C 18:1 n-6 & 1.7 & 1.5 & 1.2 \\
\hline C 20:1 n-3 & 1.7 & 1.2 & 1.7 \\
\hline Total AGMI* & $5.8^{*}$ & $5.5^{*}$ & $5.3^{*}$ \\
\hline C 18:2 n-6 & 0.8 & nd & 1.0 \\
\hline C 18:3 n-3 & 1.2 & 4.6 & 7.5 \\
\hline C 20:2 n-3 & 3.2 & 1.2 & nd \\
\hline C 20:3 n-3 & 1.4 & 1.3 & 1.3 \\
\hline C 20:5 n-3 & 3.4 & 2.7 & 2.6 \\
\hline C 22:4 n-3 & 7.0 & 7.0 & 7.7 \\
\hline C 22:5 n-3 & 4.0 & 2.2 & 2.7 \\
\hline C 22:6 n-3 & 4.2 & 8.3 & 3.9 \\
\hline Total AGPI* & $25.2 *$ & $27.3 *$ & $26.7^{*}$ \\
\hline Total AGNI & 29.2 & 20.4 & 25.9 \\
\hline
\end{tabular}

$\mathrm{N}=3$, números de réplicas (conjuntos de 60 ostras).

nd: no detectado.

Valores encontrados $=($ medias \pm 0.1$)$.

Límite de detección $\geq 0.1$.

${ }^{*} P>0.05$, no hubo diferencias significativas.

Los ácidos grasos monoinsaturados estuvieron presentes en menor proporción en todas las muestras, con valores que variaron entre $5.3 \%$ y $5.8 \%$, prevaleciendo el ácido oleico
Da Cruz (2004) revealed that the saturated fat concentration found during this research are two-fold those detected by these authors. This increment can be ascribed to an excessive fluidity in the cell membrane of the species, induced by the elevated temperatures of the study areas.

The statistical analyses performed revealed nonsignificant differences $(P>0.05)$ in the concentration of saturated, polyunsaturated and monounsaturated fatty acids of the samples examined. The high values of saturated fat in these lipids suggest that an excessive intake of these lipids might increase blood cholesterol levels, especially those of the LDL fraction. Although the mechanism for this increase is not altogether known, several epidemiological studies (Abbey et al. 1990, Roberts 1992, Cohn et al. 2002, Vakkilainen et al. 2002) have indicated that these fatty acids enrich the phospholipids of the cell membrane, thus interfering with the LDL receptors' normal function and reducing LDL absorption, hence diminishing the concentration of cholesterol. Thus, these concentrations of saturated fats in phospholipids could help reduce the formation of these extremely harmful lipoproteins.

The polyunsaturated fatty acids ranged from $5.2 \%$ to $27.3 \%$, the minimum values corresponding to the Chacopata oysters and the maximum to those from La Restinga. Adrenic acid (22:4 n-3) prevailed with an appraisal between $7.0 \%$ and $7.7 \%$, followed by docosahexaenoic acid (22:6n-3), linolenic acid (18:3 n-3) and docosapentaenoic acid (20:5 n-3). The high contribution of polyunsaturated fatty acids supplied by the oysters from the different areas under study suggests their utilization as a food source and energy depots in human beings (Satue et al. 1994)

The monounsaturated fatty acids showed lower proportions in all samples, with values ranging from $5.3 \%$ to $5.8 \%$, and oleic acid (18:3 n-3) and gadoleic acid (20:1) prevailing. The concentration of monounsaturated fatty acids was similar in all the oysters, oleic acid being the main precursor. In this sense, it can be stated that the dietary intake of these fatty acids could have an empowering effect on high density lipoproteins (HDL or "good cholesterol"), since it has already been proven that HDL reduce LDL cholesterol (or "bad cholesterol"), thus reducing the risk of cardiovascular disease (Vakkilainen et al. 2002).

The minimum values of polyunsaturated fatty acids coincided with the maximum values of saturated fatty acids present in the phospholipids and triacylglycerols, which could be related to the structural changes in the properties of the cell membranes of the species, tissues where saturated fats are stored to be subsequently used as an energy source (Stiwell 1993).

\section{Analysis of triacylglycerol fatty acids}

An appraisal of the saturated fatty acids in the triacylglycerols (table 4) showed minimum values of $22.7 \%$ in the Chacopata oysters, followed by the La Restinga and Caroni specimens, with $62.3 \%$ and $71.2 \%$, respectively, and a 
(18:1 n-3) y el gadoleico (20:1). Las concentraciones de ácidos grasos monoinsaturados fueron similares en todas las ostras, dando como principal precursor el ácido oleico. Con relación a estos ácidos grasos se puede decir que su consumo pudiera tener un efecto potenciador en las lipoproteínas de alta densidad (HDL o "colesterol bueno"), ya que se ha comprobado que la presencia de HDL disminuye el colesterol LDL (o “colesterol malo"), reduciendo así el riesgo de enfermedades cardiovasculares, tal como lo señalaron Vakkilainen et al. (2002).

Los valores mínimos de ácidos grasos poliinsaturados coinciden con los valores máximos de ácidos grasos saturados presentes en los fosfolípidos y triacilgliceroles, lo cual tendría relación con los cambios estructurales en las propiedades de las membranas celulares de la especie, donde las grasas saturadas son almacenadas en los tejidos de la ostra para luego ser utilizadas como fuente de energía (Stiwell 1993).

\section{Análisis de los ácidos grasos en los triacilgliceroles}

Los ácidos grasos saturados de los triacilgliceroles (tabla 4) mostraron valores mínimos, de $22.7 \%$, en las ostras provenientes de Chacopata, seguidas por las de La Restinga con $62.3 \%$ y las del Pantano de Caroni con 71.2\%, prevaleciendo el ácido palmítico (16:0). Los ácidos grasos poliinsaturados estuvieron entre $13.0 \%$ y $48.3 \%$, con valores mínimos en las ostras de las lagunas de La Restinga y el Pantano de Caroni, y los máximos en las muestras de Chacopata. Estos ácidos estuvieron representados principalmente por el ácido adrénico (22:4), con valores de $31.5 \%$ en $C$. rhizophorae provenientes de Chacopata y valores menores en los especimenes del Pantano de Caroni (7.7\%) y La Restinga (4.5\%).

El análisis estadístico realizado evidenció la existencia de diferencias altamente significativas $(P<0.05)$ en las concentraciones de ácidos grasos saturados, poliinsaturados y monoinsaturados en las muestras estudiadas, observándose la formación de dos grupos homogéneos (uno conformado por las muestras de Chacopata y el otro por las de La Restinga y el Pantano de Caroni) para cada concentración de grasas.

La elevada concentración de ácidos grasos saturados en los triacilgliceroles de las ostras de La Restinga y el pantano de Caroni, reflejan un acondicionamiento energético de los triacilgliceroles por parte de las gónadas para entrar en su próximo ciclo reproductivo, el cual esta influenciado específicamente por las altas temperaturas, vientos moderados y aguas fangosas de las tres zonas, que a su vez proporcionan pocos recursos alimenticios para que las especies puedan nutrirse. Por otra parte, se observan bajas concentraciones de ácidos grasos poliinsaturados, lo cual puede deberse a la degradación que éstos sufren para la formar eicosanoides. Entre estos compuestos activos fisiológica y farmacológicamente se encuentran las prostaglandinas, las cuales tienen una participación importante en el crecimiento y la reproducción de los seres vivos. Sin embargo, la elevada concentración de ácidos grasos poliinsaturados en
Tabla 4. Composición porcentual de los ácidos grasos en los triacilgliceroles de Crassostrea rhizophorae proveniente de las lagunas de Chacopata y La Restinga y el Pantano de Caroni (AGS, ácidos grasos saturados; AGMI, ácidos grasos monoinsaturados, AGPI, ácidos grasos poliinsaturados; AGNI, ácidos grasos no identificados).

Table 4. Fatty acid percentages for triacylglycerols in Crassostrea rhizophorae from Chacopata and La Restinga lagoons and Caroni Swamp (AGS, saturated fatty acids; AGMI, monounsaturated fatty acids, AGPI, polyunsaturated fatty acids; AGNI, unidentified fatty acids).

\begin{tabular}{|c|c|c|c|}
\hline Ácidos grasos & $\begin{array}{c}\text { Chacopata } \\
\text { (media } \pm 0.1 \text { ) }\end{array}$ & $\begin{array}{l}\text { La Restinga } \\
\text { (media } \pm 0.1 \text { ) }\end{array}$ & $\begin{array}{c}\text { Caroni } \\
(\text { media } \pm 0.1)\end{array}$ \\
\hline C 13:0 & 7.5 & 12.1 & 12.1 \\
\hline C 14:0 & 0.8 & 10.6 & 12.9 \\
\hline C 15:0 & nd & 1.9 & 1.6 \\
\hline C 16:0 & 3.0 & 27.1 & 32.0 \\
\hline C 17:0 & 0.7 & 0.8 & 2.3 \\
\hline C 18:0 & 0.6 & 4.4 & 5.2 \\
\hline C 19:0 & nd & 2.2 & 0.4 \\
\hline C 20:0 & nd & 0.7 & 1.5 \\
\hline C 23:0 & nd & 1.6 & 0.7 \\
\hline C 24:0 & 10.1 & 0.9 & 2.5 \\
\hline Total AGS & $22.7^{*}$ & $62.3 * *$ & $71.2^{* *}$ \\
\hline C $16: 1$ & 0.7 & 3.5 & 2.8 \\
\hline C $18: 1 \mathrm{n}-3$ & 0.6 & 2.3 & 1.6 \\
\hline C $18: 1 \mathrm{n}-6$ & nd & 2.6 & 1.9 \\
\hline C 20:1 n-3 & nd & 1.8 & 1.5 \\
\hline Total AGMI & $1.3^{*}$ & $10.2 * *$ & $7.8^{* *}$ \\
\hline C $18: 2 n-6$ & 8.9 & 0.9 & 0.6 \\
\hline C $18: 3 n-3$ & 1.0 & 1.1 & 1.7 \\
\hline C $20: 2 n-3$ & 0.8 & 2.2 & nd \\
\hline C 20:4 n-3 & 0.9 & 0.8 & nd \\
\hline C 20:5 n-3 & 0.7 & 1.7 & 1.4 \\
\hline C 22:4 n-3 & 31.5 & 4.5 & 7.7 \\
\hline C 22:5 n-3 & 4.5 & 0.4 & 1.1 \\
\hline C 22:6 n-3 & nd & 1.4 & 0.5 \\
\hline Total AGPI & $48.3^{*}$ & $13.0 * *$ & $13.0 * *$ \\
\hline Total AGNI & 27.7 & 14.5 & 8.0 \\
\hline
\end{tabular}

$\mathrm{N}=3$, números de réplicas (conjuntos de 60 ostras).

nd: no detectado.

Valores encontrados $=($ medias \pm 0.1$)$.

Límite de detección $\geq 0.1$.

* $P<0.05$, hubo diferencias altamente significativas (dos grupos homogéneos: * primer grupo $y$ ** segundo grupo).

prevalence of palmitic acid (16:0). The polyunsaturated fatty acids ranged between $13.0 \%$ and $48.3 \%$, the minimum values corresponding to the La Restinga and Caroni spat populations and the maximum to the Chacopata specimens. They were 
los triacilgliceroles de C. rhizophorae en la Laguna de Chacopata se debe a la elevada presencia de un recursos alimenticios en sus aguas (fitoplancton) aunada a las condiciones ambientales de altas temperaturas, vientos fuertes y alta concentración salina. Esto permite un mejor acondicionamiento bioquímico en las células germinales de la especie para cumplir con sus funciones gametogénicas, siendo importante la participación de estos ácidos grasos en el crecimiento de nuevos seres en formación tal como lo señalaron Kinsella (1986) y Cohn et al. (2002).

Al comparar los valores obtenidos de fosfolípidos y triacilgliceroles, con excepción de los ácidos grasos en los triacilgliceroles de las muestras provenientes de la Laguna de Chacopata, se constató que los fosfolípidos presentaron mayores porcentajes de ácidos grasos poliinsaturados en comparación con el observado para los triacilgliceroles. Estos resultados son razonables, ya que los fosfolípidos están constituidos básicamente por ácidos grasos poliinsaturados en su estructura, permitiendo así el flujo a través de las membranas celulares de la especie (Carmena 1986).

La presencia de los ácidos grasos 20:5 n-3, 22:5 n-3 y 22:6 n-3 en los triacilgliceroles puede ser debido a que aquellos son sintetizados a partir del ácido linolénico (18:3) mediante desaturaciones y elongaciones sucesivas. Adicionalmente, estos ácidos grasos son esenciales para que la especie pueda cumplir con sus funciones reproductivas, asegurando así los recursos energéticos y nutricionales para el desarrollo y crecimiento de los nuevos organismos; sin embargo, los organisos marinos son incapaces de sintetizarlos, por tanto su presencia en estas muestras se debe a su obtención a través de la dieta (Villarroel 1997, Tineo 2002).

\section{Agradecimientos}

Los autores desean expresar su agradecimiento al Consejo de Investigación de la Universidad de Oriente y al Departamento de Química (Núcleo de Sucre) por haber financiado parcialmente esta investigación. Al Instituto Universitario de Tecnología-Cumaná, por haber facilitado la utilización de los cromatógrafos de gases. A Luisa Rojas y la Universidad de las Indias Occidentales (Trinidad y Tobago) por la recolección de las muestras en el Pantano de Caroni.

\section{Referencias}

Abbey M, Clifton M, McMurchie J, Nestel J. 1990. Effect of a high fat cholesterol diet with or without eicosapentanoic acid on plasma lipids, lipoproteins and lipid transfer protein activity in the marmoset. Atherosclerosis 81: 163-174.

Bonilla J. 1972. Variación mensual del compuesto químico en el ostión de mangle y el ostión cultivado. Bol. Inst. Oceanogr. Univ. Oriente 11(2): 115-120.

Bonilla J. 1975. Variación mensual de la composición química del ostión de mangle en la Laguna Las Maritas (Venezuela). Bol. Inst. Oceanogr. Univ. Oriente 14(1): 117-127.

Bonilla J, Benitez J, Okuda T. 1969. Variación estacional de la composición química del ostión Crassostrea rhizophorae mainly represented by adrenic acid (22:4), with values of $31.5 \%, 7.7 \%$ and $4.5 \%$ in C. rhizophorae from Chacopata, Caroni and La Restinga, respectively.

The statistical analysis reflected highly significant differences $(P<0.05)$ in the concentrations of saturated, polyunsaturated and monounsaturated fatty acids of the samples under study, two homogeneous groups becoming manifest for each fat concentration, one consisting of the Chacopata samples and the other of the La Restinga and Caroni samples.

The elevated concentration of saturated fatty acids in the triacylglycerols from the La Restinga and Caroni oysters reflects an energy conditioning by the gonads to enter their next reproductive cycle. This conditioning is specifically influenced by the high temperatures, moderate winds and muddy waters of these areas, which supply scant food resources for the nourishment of the species. On the other hand, the concentration of polyunsaturated fatty acids was low, possibly due to the degradation undergone by these fatty acids to form eicosanoids, active physiological and pharmacological compounds among which prostaglandins have an important participation in the growth and reproduction of all living beings. However, the high concentration of polyunsaturated fatty acids in the triacylglycerols of the Chacopata oysters is due to the abundance of food in the lagoon's waters (phytoplankton), along with the high temperatures, strong winds and high salt concentration, a concurrence of stimuli allowing a better biochemical conditioning by the germ cells of the species to fulfill their gametogenic functions, the participation of these fatty acids being important for offspring formation, as pointed out by Kinsella (1986) and Cohn et al. (2002).

A comparison of the phospholipid and triacylglycerol values, excepting the Chacopata triacylglycerol fatty acids, indicates a greater percentage of phospholipid polyunsaturated fatty acids. These results are logical, as phospholipids are basically structured by polyunsaturated fatty acids, thus permitting the fluidity of the cell membranes of the species (Carmena 1986).

The presence of 20:5, 22:5 and 22:6, all n-3 fatty acids, in the triacylglycerols can be attributed to the fact that they are synthesized from linolenic acid through successive desaturation and chain elongation. These fatty acids are essential for the species to fulfill its reproductive functions and to safeguard the nourishment and energy resources necessary for the development of offspring. Marine creatures, however, are incapable of synthesizing them, and their presence in these samples is due to their being assimilated through the diet (Villarroel 1997, Tineo 2002).

\section{Acknowledgements}

The authors thank the Research Council and Chemistry Department (Sucre Campus) of Oriente University for financial support of this research, and the Instituto Universitario de Tecnología-Cumaná for the use of the gas chromatograph. We thank L Rojas and the University of the West Indies (Trinidad) for providing the Caroni Swamp samples. 
(Guilding) en Laguna Grande y la Bahía de Mochima. Bol. Inst. Oceanogr. Univ. Oriente 8 (1-2): 46-52.

Cardona R, Soltero Y. 1987. Aterosclerosis al día. Tribuna Médica Venezolana C.A. 1: 578.

Carmena A. 1986. Temas de Actualización sobre Colesterol, Lipoproteínas y Aterosclerosis. Laboratorios Servir, SA, Argentina, 88 pp.

Carter L. 1993. Analysis of Triglycerides. Academic Press, New York, $32 \mathrm{pp}$.

Cavalcanti R, Da Cruz G. 2004. Proximate composition and fatty acid content of the mangrove oyster Crassostrea rhizophorae along the year seasons. Braz. Arch. Biol. Technol. 47(6): 955-960.

Cohn J, Tremblay R, Batal R, Jacques H, Veilleux L, Rodriguez C, Bernier L, Mamer O, Davignon J. 2002. Plasma kinetics of VLDL and HDL apoC-I in normolipidemic and hypertriglyceridemic subjects. J. Lipid Res. 43(10): 1680-1687.

Cristie W. 1973. Lipids Analysis. 1st ed. Pergamon Press, New York, $78 \mathrm{pp}$.

Establier R. 1966. Variación estacional de la composición química del ostión Crassostrea rhizophorae y su relación en las variaciones hidrológicas y de fitoplancton. Invest. Pesq. (30): 501-528.

Farnworth E, Thompson B, Kramer J. 1982. Quantitative determination of neutral lipids on chrome rods. J. Chromatogr. 264: 463-747.

Frías J. 1976. La Salinidad. Factor abiótico a considerar en las principales funciones del ostión como objeto de cultivo. Contribuciones. Ministerio de la Industria Pesquera, 16 pp.

Goldbourt V, Yaari S. 1990. Cholesterol and coronary heart disease mortality. Aterosclerosis 10(4): 512-519.

Kinsella J. 1986. Dietary fish oils. Nutrition Today 21(6): 5-14.

Martino R, Cyrino J, Portz L, Trugo L. 2002. Performance and fatty acid composition of surubim (Pseudoplatystoma coruscans) fed diets with animal and plant lipids. Aquaculture 209: 233-246.

Maza O. 1991. Caracterización de lípidos en el tejido muscular de diferentes especies de peces de origen marino en el oriente del país. Trabajo de pregrado, Departamento de Química, Universidad de Oriente, Cumaná, Venezuela, 35 pp.

Noffs M. 2002. Composición centesimal de ácidos grasos en algunas especies de peces marinos, verificación, utilización y obtención de sus altos valores de ácidos grasos poliinsaturados omega 3. Tesis de maestría, Universidad de San Francisco, Braganca Paulista, SP, Brazil.

Overturf M, Dryer R. 1967. Experiments in Physiology and Biochemistry. Vol. 2. Academic Press, New York, 1976 pp.

Pieter D, Rietveld C, Swart R. 1994. Effect of dietary fish oil on blood levels of free fatty acids, ketone bodies and triacylglycerol in humans. Lipids 29: 41-45.

Roberts C. 1992. Atherosclerotic risk factors, are there ten or is there only one? Atherosclerosis 97: S5-S9.

Satue M, López M, Agramont A. 1994. Fatty acid composition of trout oil. Food Chem. 50: 363-365.

Stiwell W. 1993. Docosahexanoic acid increase permeability of lipid vesicles and tumor cell. Lipids 28: 103-108.

Tineo C. 2002. Diversidad de los moluscos en la Laguna de Bocaripo, Guayacán, Estado Sucre, Venezuela. Trabajo de pregrado, Departamento de Biología, Universidad de Oriente, Cumaná, Venezuela, 49 pp.

Vakkilainen J, Jauhiainen M, Ylitalo K, Nuotio I, Viikari J, Ehnholm C, Taskinen M. 2002. LDL Particle size in familial combined hyperlipidemia: Effects of serum lipids, lipoprotein-modifying enzymes, and lipid transfer proteins. J. Lipid Res. 43(4): 598-603.

Villarroel A. 1997. Caracterización y cuantificación de las diferentes clases de lípidos presentes en el hígado y tejido muscular del marao (Tylosurus crocodilus). Trabajo de pregrado, Departamento de Química, Universidad de Oriente, Cumaná, Venezuela, 73 pp.

Recibido en marzo de 2005;

aceptado en abril de 2006. 